\title{
Study of Internal Pressure and Related Parameters in Ternary Liquid Mixtures Using Ultrasonic Velocity Measurement
}

\author{
P. Paul Divakar ${ }^{1 *}$ and K. Samatha ${ }^{2}$ \\ Department of Physics, Sir. C. R. Reddy (A) College, Eluru-534007, India \\ Department of Physics, Andhra University, Visakhapatnam-533003, India \\ putla_paul@yahoo.co.in
}

\begin{abstract}
Ultrasonic velocity( $(U)$, density $(\rho)$ and viscosity $(\eta)$ measurements have been taken in two ternary liquid mixtures Ethylene glycol + cyclohexanone + Isopropyl acetate and Ethylene glycol + cyclohexanone + Isobutyl acetate with fixed mole fraction (0.4) of cyclohexanone, at different temperatures with increasing mole fraction of ethylene glycol. The experimental data has been used to calculate the free volume, molar volume, internal pressure, enthalpy, Gibbs free energy, Molar sound velocity and their excess values. They have been analysed to discuss the nature and strength of intermolecular interactions in these mixtures. The compositional variation of excess parameters have been correlated using the Redlich-Kister equation.
\end{abstract}

Keywords: Ternary mixtures, internal pressure, molar sound velocity, excess parameters intermolecular interactions

\section{Introduction}

The study of thermodynamic properties of binary and ternary mixtures contributes to an understanding of the behavior of different liquids and functional groups. Number of studies on the thermodynamic properties of ternary mixtures has increased in recent years due to industrial applications[1-5]. A third component added to a mixture of two immiscible components brings about the phenomenon in certain ternary liquid mixtures has been studied earlier [6-9] by observing on the distribution of molecules of one component between the other two components and the formation of hitherto unknown compounds from a study of the change in free volume, molar volume, internal pressure and other thermodynamic parameters.

The measurement of ultrasonic velocity has been adequately employed in understanding the molecular interactions in liquid mixtures. Molecular interaction studies can be carried out by both spectroscopic and non-spectroscopic techniques. However, ultrasonic velocity and viscosity measurements have been widely used in the field of interactions and structural aspect evaluation studies.

Internal pressure has gained significant interest by chemists, physicists and chemical engineers in past, as it provides a measure of explaining molecular interactions, internal structure, clustering phenomenon and dipolar interactions. Internal pressure has been a subject of active interest among several researchers during recent past [7-10]. Several attempts have been made by a number of investigators [11-15] to calculate the internal pressure of liquids and liquid mixtures theoretically.

The measurement of internal pressure is important in the study of the thermodynamic properties of liquids. The internal pressure is the cohesive force, which is a resultant of force of attraction and force of repulsion between the molecules. Cohesion creates pressure within the liquid. So internal pressure gives an idea of the solubility characteristics. Dissolved solutes exist under the internal pressure of the medium and their interactions with the solvent arise through hydrogen bonding, charge transfer, Columbic (or) Vanderwaal's interaction. 


\section{Experimental Details}

Ultrasonic velocity (U), measurements were made with a single crystal variable path ultrasonic interferometer (Model F-81; Mittal enterprises) at $2 \mathrm{MHz}$ frequency with an accuracy of $\pm 0.01 \%$. The densities ( $\rho$ ) were measured using specific gravity bottles by relative measurement method with accuracy of $\pm 0.01 \mathrm{kgm}^{-3}$ and compared at the working temperature with the corresponding literature values. The viscosity $(\eta)$ measurements were made with Ostwald viscometer with an accuracy of $\pm 0.001 \mathrm{Nsm}^{-2}$.

The chemicals used in the present work are AR grade of purity $>99 \%$ from E-Merck and Hi-Media and they were used without further purification.

1. Monoethylene glycol + Cyclohexanone + Isopropyl acetate (MEG+CY+IPA)

2. Monoethylene glycol + Cyclohexanone + Isobutyl acetate (MEG+CY+IBA).

Actually monoetyhlene glycol and isopropyl acetate/ isobutyl acetate liquids are immiscible and by the addition of cyclohexanone only the ternary mixtures are formed. In all the four systems, the mole fraction of the second component, cyclohexanone $(\mathrm{CY})$ is kept fixed at $0.4\left(\mathrm{X}_{2}\right)$, while the mole fractions of the remaining two have been varied from 0.0 to $0.6\left(\mathrm{X}_{1}\right.$ and $\left.\mathrm{X}_{3}\right)$ so as to have the mixtures of different compositions. There is no significance in fixing mole fraction of the second liquid component $(\mathrm{CY})$ at $\mathrm{X}_{2}=0.4$.

\section{Theoretical Aspects}

\subsection{Molar Volume of the Liquid Mixture $\left(V_{m}\right)$}

The molar volumes of the mixtures have been computed using its relationship with the molecular weight and mole fraction of the constituent is given below

$$
V_{m}=\frac{M_{e f f}}{\rho_{m i x}}
$$

Where $M_{\text {eff }}=\frac{M_{1} X_{1}+M_{2} X_{2}+M_{3} X_{3}}{X_{1}+X_{2}+X_{3}}$ here $\mathrm{M}_{1}, \mathrm{M}_{2}, \mathrm{M}_{3}$ are the molecular weights of corresponding liquids and $X_{1}, X_{2}, X_{3}$ are their mole fractions.

\subsection{Free Volume $\left(V_{f}\right)$}

The free volumes of the mixtures have been computed using its relationship with the ultrasonic velocity and viscosity is given below

$V_{f}=\left(\frac{M_{e f f} U}{k \eta}\right)^{3 / 2}$

where $\mathrm{k}$ is a constant, independent of temperature and it's value is $4.28 \mathrm{X} 10^{9}$ for all liquids.

\subsection{Internal Pressure $\left(\pi_{\mathbf{i}}\right)$}

On the basis of dimensional analysis, using free volume concept, the following expression can be used for calculating internal pressure

$$
\pi_{i}=b R T\left(\frac{k \eta}{U}\right)^{1 / 2} \frac{\rho^{2 / 3}}{M_{e f f}^{7 / 6}}
$$

here $\mathrm{b}$ is packing factor ( $\mathrm{b}=2), \mathrm{k}$ is a constant, independent of temperature and it's value is $4.28 \times 10^{9}$ for all liquids, $\mathrm{R}$ is universal gas constant and $\mathrm{T}$ is absolute temperature. 


\subsection{Enthalpy ( H )}

The enthalpy of the liquid mixture is given by

$H=\pi_{i} V_{m}$

\subsection{Gibb's Free Energy for Activation of Flow $(\Delta G)$}

The Gibb's free energy for activation of flow $(\Delta \mathbf{G})$ can be calculated using relation

$\Delta G=R T \ln \left(\eta V_{m}\right)$

where $\mathrm{R}$ is the Universal gas constant $\left(8.31432 \times 10^{7} \mathrm{JK}^{-1}\right)$, and $\mathrm{T}$ is the absolute temperature

\subsection{Molar Sound Velocity or Rao's Constant (R)}

Rao's constant can be calculated by the relation

$R=V_{m} U^{1 / 3}$

\subsection{Excess Thermodynamic Parameters}

The excess thermodynamic functions ( $\mathrm{Y}^{\mathrm{E}}$ ) provide a way to represent directly the deviation of a solution from ideal behaviour. The difference between the thermodynamic function of mixing for a real system and the value corresponding to a perfect solution at the same temperature, pressure and composition is called the thermodynamic excess function, denoted by $\mathrm{Y}^{\mathrm{E}}$. Excess values for all the parameters are computed using the general formula

$Y^{E}=Y_{\text {exp }}-\left(X_{1} Y_{1}+X_{2} Y_{2}+X_{3} Y_{3}\right)$

The excess properties $\mathrm{Y}^{\mathrm{E}}$ were fitted by non linear least square method to Redlich Kister type polynomial

$Y^{E}=x_{1}\left(1-x_{1}\right) \sum_{i=0}^{p} A_{i}\left(2 x_{1}-1\right)^{i}$

In each case, the optimum number of coefficients $A_{i}$ was determined from an examination of the variation of standard deviation $(\sigma)$ as calculated by

$\sigma\left(Y^{E}\right)=\left\lfloor\frac{\sum\left(Y_{\text {exp }}^{E}-Y_{c a l}^{E}\right)}{(n-p)}\right\rfloor^{\frac{1}{2}}$

where ' $n$ ' represents the number of experimental points and ' $p$ ' is the number of coefficients.

\section{Results and Discussions}

The two ternary systems contain an aliphatic cyclic compound i.e., cyclohexanone along with straight or branch chained liquids. An exhaustive survey of literature reveals that a few researchers studied the ternary mixtures using ultrasonics. Hence a systematic study has been taken up for two ternary mixtures at four temperatures 303, 308, 313 and $318 \mathrm{~K}$.

The measured values of density, ultrasonic velocity, viscosity and the evaluated parameters are presented in Table 1.1 for the ternary system-I and Table 2.1 for the ternary system-II. From tables it can be noticed that, at all the four temperatures, the values of density $(\rho)$, ultrasonic velocity $(U)$ and viscosity $(\eta)$ increase with increase in mole fraction of monoethylene glycol. This type of trend is observed by S Thirumaran et. 
al., [16] for the ternary mixtures they studied. It is evident that the pronounced increase or decrease in these parameters with composition of mixtures indicates the presence of interactions between the component molecules in the ternary mixtures. It can also be observed that the ultrasonic velocity is increasing with increase in mole fraction of monoethylene glycol and is decreasing with increase in temperature, for the ternary systems under study. This trend indicates specific interactions among the constituents of the mixtures. This behaviour at such concentrations which is different from the ideal mixture behaviour can be attributed to intermolecular interactions $[17,18]$. The chemical interaction may involve the association due to hydrogen bonding or due to dipole-dipole interaction or may be due to the formation of charge-transfer complexes. All these processes may lead to strong interaction forces [19]. The increase in velocity in these liquid mixtures suggest that molecular interactions among the molecules of the components of liquid mixture.

An analysis of the viscosity values from the tables it can be observed that the viscosity is in increasing trend with increase in mole fraction of monoethylene glycol and is decreasing with increase of temperature. Similar trend is also observed for the density values. This kind of non-linearity indicates the presence of molecular interactions.

It can be observed that the values of molar volume $(\mathrm{Vm})$ decreases with increase in concentration of MEG in both the ternary liquid systems. This is because of the fact, that molecular weight is directly proportional to the molar volume. The molecular weights of components of the mixtures are IPA-102.13, IBA-116.16 (alkyl acetates), CY-98.15, MEG-62.07. Since alkyl acetates have higher molecular weights in the solution comparing with others, whose concentration is decreasing in the mixture supports the calculation. Moreover, molar volume $(\mathrm{Vm})$ also increases with rise in temperature in the

present study, which probably from the fact that thermal energy facilitates an increase in the molecular separation in the liquid mixtures and thus leads to an increase in molar volume $\left(\mathrm{V}_{\mathrm{m}}\right)$ with increase of temperature [20].

It is observed that for the ternary liquid systems, the free volume $\left(\mathrm{V}_{\mathrm{f}}\right)$ decrease and the internal pressure $\left(\pi_{i}\right)$ increase with increase in concentration of MEG. Further, the decrease in free volume and increase in internal pressure with rise in concentration of MEG in all the systems clearly show the increasing magnitude of interactions. Similar trend was observed by R Thyagarajan et al., [21] also supports the present discussion.

Here the variation of enthalpy $(\mathrm{H})$ with mole fraction of MEG follows the same trend as that of the internal pressure. The increase in enthalpy with the increase in MEG and also with increase in temperature suggests specific interactions among the molecules of the liquid mixtures.

The Gibb's free energy of activation flow $(\Delta \mathrm{G})$ for the ternary mixtures under study are shown in the tables, form which it is inferred that the values of $\Delta G$ are negative. The decrease in Gibb's free energy in all the systems indicates that, the need for shorter time for the cooperative process or the rearrangement of the molecules in the mixtures.

Rao's constant (R) constant show a decreasing trend with increase of mole fraction of MEG as can be seen from Figure 1.3, Figure 2.3. More over in both the cases the values increase with increase in temperature. These non-linear trends may be attributed to possibility of molecular interactions that are taking place.

\subsection{Excess Acoustical and Thermodynamic Parameters}

In order to understand the nature of molecular interactions between the components of the liquid mixtures, it is of interest to discuss the same in terms of excess parameters rather than actual values. Non-ideal liquid mixtures show considerable deviation from linearity in their concentrations and this can be interpreted as the presence of strong or weak interactions. 
Table 1.1. Ternary Liquid Mixture - I: MEG +CY + IPA Density, Ultrasonic Velocity, Viscosity and Related Parameters at Temperatures $303,308,313$ and $318 \mathrm{~K}$

\begin{tabular}{|c|c|c|c|c|c|c|c|c|c|c|}
\hline $\begin{array}{c}\mathbf{M E} \\
\mathbf{G} \\
\mathbf{X}_{1}\end{array}$ & $\begin{array}{c}\text { IPA } \\
\mathbf{X}_{3}\end{array}$ & $\begin{array}{c}\rho \\
\mathbf{K g m}^{-3}\end{array}$ & $\underset{\mathrm{ms}^{-1}}{\mathrm{U}}$ & $\begin{array}{c}\eta \mathbf{x} \\
10^{-3} \\
\mathrm{Ns} / \mathrm{m}^{2}\end{array}$ & $\begin{array}{c}\mathrm{V}_{\mathrm{m}} \\
\times \mathbf{x}^{-5} \\
\mathrm{~m}^{3} / \mathrm{mol}\end{array}$ & $\begin{array}{c}V_{\mathrm{f}} \\
\mathbf{x} \mathbf{1 0}^{-8} \\
\mathrm{~m}^{3} / \mathbf{m o l}\end{array}$ & $\begin{array}{c}\pi_{\mathrm{i}} \\
\times \mathbf{1 0}{ }^{16} \\
\mathbf{N} / \mathbf{m}^{2}\end{array}$ & $\begin{array}{c}\text { H } \\
\times 1^{12} \\
\text { Joule/ } \\
\text { mol }\end{array}$ & $\begin{array}{c}\Delta G \\
\times 10^{11} \\
\text { Joule/ } \\
\text { mol }\end{array}$ & $\begin{array}{c}\mathbf{R} \\
\times 10^{-7} \\
\mathbf{m}^{3} / \mathbf{m o l} \\
(\mathbf{m} / \mathbf{s})^{1 / 3}\end{array}$ \\
\hline \multicolumn{11}{|c|}{ 303K } \\
\hline 0.000 & & & & 0.6652 & & & 3.3594 & 3.8407 & -4.13 & 2.564 \\
\hline 0.108 & & & & 0.7502 & & 25.848 & 3.7383 & 4.0119 & -4.11 & 2.391 \\
\hline 0.194 & & & & 0.8553 & 10.731 & 21.289 & 4.1632 & 4.2157 & -4.09 & 2.234 \\
\hline 0.267 & 0.4 & & & 0.9712 & $\begin{array}{l}10.126 \\
9.6327\end{array}$ & & 4.5954 & 4.4267 & -4.07 & 2.109 \\
\hline 0.379 & 0.3 & $\begin{array}{l}93 \\
05\end{array}$ & & 1.2620 & $\begin{array}{l}9.6327 \\
88026\end{array}$ & 224 & 5.5233 & 4.9117 & -4.03 & 1.928 \\
\hline 0.423 & 0.2 & 95 & & 1.4620 & $\begin{array}{l}8.8926 \\
85705\end{array}$ & & 6.0780 & 5.2147 & -4.00 & 1.850 \\
\hline 0.497 & 0.1 & $\begin{array}{l}97 \\
09\end{array}$ & & 2.0640 & $\begin{array}{l}8.5795 \\
8.0705\end{array}$ & $\begin{array}{l}7.7502 \\
7.7180\end{array}$ & 7.4701 & 6.0287 & -3.93 & 1.726 \\
\hline 0.527 & 0.103 & 99 & & 2.5830 & 8.0705 & & 8.4850 & 6.6545 & -3.88 & 1.669 \\
\hline 0.553 & 0.073 & 101 & 1463.2 & 3.3571 & 7.8427 & 092 & 9.7948 & 7.4896 & -3.82 & 1.622 \\
\hline 0.578 & 0.046 & 1024.8 & 1496.4 & 4.9421 & 7.6464 & 2.3314 & 12.034 & 8.9822 & -3.73 & 1.576 \\
\hline 0.600 & $\begin{array}{l}0.021 \\
0.000\end{array}$ & $\begin{array}{l}1036.7 \\
1049.5\end{array}$ & $\begin{array}{l}1526.3 \\
1559.6\end{array}$ & 10.190 & $\begin{array}{l}7.4636 \\
7.2899\end{array}$ & $\begin{array}{l}1.3193 \\
0.4525\end{array}$ & 17.464 & 12.731 & -3.55 & 1.533 \\
\hline \multicolumn{11}{|c|}{$308 K$} \\
\hline 0.000 & & & & 0.6210 & 11.741 & & 3.3279 & 3.8404 & -4.21 & 1.406 \\
\hline 0.108 & 0.600 & 871.2 & 1114.8 & 0.6981 & 11.061 & 33.277 & 3.6874 & 4.0019 & -4.20 & 1.324 \\
\hline 0.194 & 0.492 & 88 & 1158.6 & 0.7890 & 10.471 & 28.224 & 4.0761 & 4.1851 & -4.18 & 1.246 \\
\hline 0.267 & 0.405 & 90 & 1197.4 & 0.8940 & 10.001 & 4.034 & 4.4803 & 4.3858 & -4.16 & 1.186 \\
\hline 0.379 & 0.333 & $91^{\circ}$ & 1234.6 & 1.1441 & 9.2804 & 0.463 & 5.3307 & 4.8279 & -4.12 & 1.097 \\
\hline 0.423 & 0.220 & 942 & 1303.5 & 1.3030 & 8.9672 & 5.032 & 5.8091 & 5.0836 & -4.09 & 1.055 \\
\hline 0.497 & 0.176 & 95 & 1336.6 & 1.8071 & 8.4608 & 12.766 & 7.0579 & 5.8172 & -4.02 & 0.990 \\
\hline 0.527 & 0.103 & 978 & 1409.2 & 2.2050 & 8.2396 & 8.7504 & 7.8949 & 6.3375 & -3.98 & 0.962 \\
\hline 0.553 & 0.073 & 98 & 1445.2 & 2.7551 & 8.0460 & 7.0800 & 8.9394 & 7.0022 & -3.93 & 0.937 \\
\hline 0.578 & 0.046 & 1000.4 & 1475.2 & 3.7220 & 7.8721 & 5.4381 & 10.504 & 8.0526 & -3.86 & 0.913 \\
\hline 0.600 & $\begin{array}{l}0.021 \\
0.000\end{array}$ & $\begin{array}{l}1009.3 \\
1018.8\end{array}$ & $\begin{array}{l}1504.4 \\
1532.2\end{array}$ & 8.7931 & 7.7187 & $\begin{array}{l}3.9115 \\
1.6261\end{array}$ & 16.308 & 12.247 & -3.64 & 0.892 \\
\hline \multicolumn{11}{|c|}{ 313K } \\
\hline 0.000 & & & & 0.5530 & 11.629 & 31.808 & 3.2017 & 3.7234 & -4.31 & 1.387 \\
\hline 0.108 & & & & 0.6181 & 10.954 & 26.872 & 3.5246 & 3.8609 & -4.29 & 1.304 \\
\hline 0.194 & & & & 0.6920 & 10.368 & 22.556 & 3.8759 & 4.0187 & -4.28 & 1.226 \\
\hline 0.267 & 0.4 & & & 0.7780 & 9.8969 & 18.943 & 4.2376 & 4.1939 & -4.26 & 1.165 \\
\hline 0.379 & 0.3 & & & 0.9791 & 9.1648 & 13.564 & 4.9856 & 4.5692 & -4.22 & 1.073 \\
\hline 0.423 & 0.220 & 93 & & 1.1021 & 8.8550 & 11.404 & 5.4048 & 4.7860 & -4.20 & 1.031 \\
\hline 0.497 & 0.176 & 94. & & 1.4860 & 8.3547 & 7.4678 & 6.4702 & 5.4057 & -4.14 & 0.967 \\
\hline 0.527 & 0.103 & 96. & 134 & 1.7621 & 8.1283 & 5.8800 & 7.1364 & 5.8007 & -4.10 & 0.938 \\
\hline 0.553 & 0.073 & $\begin{array}{l}977 \\
087\end{array}$ & $\begin{array}{l}1435.5 \\
1467\end{array}$ & 2.1330 & 7.9344 & 4.4692 & 7.9467 & 6.3052 & -4.05 & 0.912 \\
\hline 0.578 & $\begin{array}{l}0.046 \\
0.021\end{array}$ & $\begin{array}{l}987.6 \\
0966\end{array}$ & $\begin{array}{l}1467.2 \\
14952\end{array}$ & 2.8112 & 7.7639 & 2.9819 & 9.2269 & 7.1637 & -3.99 & 0.890 \\
\hline 0.600 & $\begin{array}{l}0.021 \\
0.000\end{array}$ & $\begin{array}{c}996.6 \\
1008.3\end{array}$ & $\begin{array}{l}1495.2 \\
1518.4\end{array}$ & 5.7222 & 7.5878 & 1.0331 & 13.339 & 10.121 & -3.81 & 0.864 \\
\hline \multicolumn{11}{|c|}{$318 K$} \\
\hline 0.000 & & & & 0.5281 & & & 3.1839 & 0.3512 & -4.38 & 1.406 \\
\hline 0.108 & & & & 0.5901 & & $\begin{array}{l}53.211 \\
28.224\end{array}$ & 3.5000 & 0.2110 & -4.37 & 1.324 \\
\hline 0.194 & & & $\begin{array}{l}1129.6 \\
1170.4\end{array}$ & 0.6560 & & $\begin{array}{l}28.2 \angle 4 \\
24.034\end{array}$ & 3.8300 & 0.1138 & -4.36 & 1.246 \\
\hline 0.267 & & $\begin{array}{l}885.6 \\
898.4\end{array}$ & $\begin{array}{l}11 / 0.4 \\
1210.8\end{array}$ & 0.7320 & & & 4.1665 & 0.0410 & -4.34 & 1.186 \\
\hline 0.379 & & $\begin{array}{l}898.4 \\
919.5\end{array}$ & 1286.3 & 0.9070 & & & 4.8539 & -0.058 & -4.30 & 1.097 \\
\hline 0.423 & & 931.8 & 1318.2 & 1.0150 & & & 5.2443 & -0.093 & -4.28 & 1.055 \\
\hline 0.497 & & 953.2 & 1389.2 & 1.3281 & & & 6.1830 & -0.147 & -4.23 & 0.990 \\
\hline 0.527 & $\begin{array}{l}0.103 \\
0.073\end{array}$ & 964.1 & 1428.3 & 1.5490 & & & 6.7536 & -0.100 & -4.19 & 0.962 \\
\hline 0.553 & 0.046 & 973.9 & 1459.8 & 1.8621 & & & 7.4922 & -0.185 & -4.15 & 0.937 \\
\hline 0.578 & 0.021 & 982.9 & 1483.2 & 2.3272 & $\begin{array}{l}8.0400 \\
7.8721\end{array}$ & $\begin{array}{l}5.4381 \\
3.9115\end{array}$ & 8.4848 & -0.201 & -4.10 & 0.913 \\
\hline 0.600 & $\begin{array}{l}0.021 \\
0.000\end{array}$ & $\begin{array}{l}902.9 \\
991.2\end{array}$ & $\begin{array}{l}1485.2 \\
1506.3\end{array}$ & 4.1952 & 7.7187 & $\begin{array}{l}5.9115 \\
1.6261\end{array}$ & 11.518 & -0.215 & -3.95 & 0.892 \\
\hline
\end{tabular}


Table 2.1. Ternary Liquid Mixture - II: MEG +CY + IBA Density, Ultrasonic Velocity, Viscosity and Related Parameters at Temperatures $303,308,313$ and $318 \mathrm{~K}$

\begin{tabular}{|c|c|c|c|c|c|c|c|c|c|c|}
\hline $\begin{array}{c}\text { ME } \\
\mathbf{G} \\
\mathbf{X}_{1}\end{array}$ & $\begin{array}{c}\text { IBAA } \\
\mathbf{X}_{3}\end{array}$ & $\underset{\mathrm{Kgm}^{-3}}{\rho}$ & $\begin{array}{c}\mathrm{U} \\
\mathrm{ms}^{-1}\end{array}$ & $\begin{array}{c}\eta \mathbf{x} \\
\mathbf{1 0}^{-3} \\
\mathrm{Ns} / \mathrm{m}^{2}\end{array}$ & $\begin{array}{c}V_{\mathrm{m}} \\
\times \mathbf{m o}^{-5} \\
\mathrm{~m}^{3} / \mathbf{m o l}\end{array}$ & $\begin{array}{c}V_{f} \\
\times 10^{-8} \\
\mathrm{~m}^{3} / \mathrm{mol}\end{array}$ & $\begin{array}{c}\pi_{\mathrm{i}} \\
\times \mathbf{1 0} \\
\mathrm{N} / \mathrm{m}^{2}\end{array}$ & $\begin{array}{c}\text { H } \\
\times 10^{12} \\
\text { Joule/ } \\
\text { mol }\end{array}$ & $\begin{array}{c}\Delta G \\
\times 10^{11} \\
\text { Joule/ } \\
\text { mol }\end{array}$ & $\begin{array}{c}\mathbf{R} \\
\times \mathbf{1 0}^{-7} \\
\mathbf{m}^{\mathbf{3}} / \mathbf{m o l} \\
(\mathbf{m} / \mathbf{s})^{1 / 3}\end{array}$ \\
\hline \multicolumn{11}{|c|}{ 303K } \\
\hline $\begin{array}{l}0.000 \\
0.096 \\
0.248 \\
0.308 \\
0.360 \\
0.407 \\
0.479 \\
0.517 \\
0.548 \\
0.574 \\
0.600 \\
\end{array}$ & $\begin{array}{l}0.600 \\
0.503 \\
0.352 \\
0.291 \\
0.239 \\
0.192 \\
0.120 \\
0.082 \\
0.051 \\
0.025 \\
0.000\end{array}$ & $\begin{array}{c}875.4 \\
891.6 \\
922.8 \\
938.2 \\
953.3 \\
967.5 \\
992.5 \\
1006.9 \\
1020.8 \\
1034.7 \\
1049.5\end{array}$ & $\begin{array}{l}1195.6 \\
1206.8 \\
1251.7 \\
1283.5 \\
1323.2 \\
1363.2 \\
1430.6 \\
1468.4 \\
1497.6 \\
1527.4 \\
1559.6\end{array}$ & $\begin{array}{l}0.7252 \\
0.7823 \\
0.9405 \\
1.0530 \\
1.2020 \\
1.3920 \\
1.9221 \\
2.4830 \\
3.2571 \\
4.6420 \\
10.190\end{array}$ & $\begin{array}{l}12.447 \\
11.633 \\
10.354 \\
9.8335 \\
9.3828 \\
8.9839 \\
8.3624 \\
8.0388 \\
7.7682 \\
7.5265 \\
7.2893 \\
\end{array}$ & $\begin{array}{l}27.194 \\
22.857 \\
16.196 \\
13.467 \\
11.034 \\
8.8693 \\
5.4837 \\
3.7402 \\
2.4865 \\
1.4649 \\
0.4524\end{array}$ & $\begin{array}{l}3.1210 \\
3.4597 \\
4.1938 \\
4.6161 \\
5.0898 \\
5.6351 \\
6.9385 \\
8.0925 \\
9.4863 \\
11.557 \\
17.465\end{array}$ & $\begin{array}{l}3.8849 \\
4.0247 \\
4.3426 \\
4.5392 \\
4.7757 \\
5.0625 \\
5.8022 \\
6.5054 \\
7.3692 \\
8.6984 \\
12.731\end{array}$ & $\begin{array}{l}-4.088 \\
-4.086 \\
-4.069 \\
-4.053 \\
-4.032 \\
-4.006 \\
-3.942 \\
-3.888 \\
-3.828 \\
-3.747 \\
-3.557 \\
\end{array}$ & $\begin{array}{l}1.509 \\
1.389 \\
1.209 \\
1.139 \\
1.080 \\
1.029 \\
0.949 \\
0.907 \\
0.870 \\
0.837 \\
0.805\end{array}$ \\
\hline \multicolumn{11}{|c|}{ 308K } \\
\hline $\begin{array}{l}0.000 \\
0.096 \\
0.248 \\
0.308 \\
0.360 \\
0.407 \\
0.479 \\
0.517 \\
0.548 \\
0.574 \\
0.600 \\
\end{array}$ & $\begin{array}{l}0.600 \\
0.503 \\
0.352 \\
0.291 \\
0.239 \\
0.192 \\
0.120 \\
0.082 \\
0.051 \\
0.025 \\
0.000\end{array}$ & $\begin{array}{c}869.2 \\
883.6 \\
911.2 \\
923.9 \\
936.2 \\
948.8 \\
971.5 \\
985.6 \\
997.4 \\
1008.3 \\
1018.8 \\
\end{array}$ & $\begin{array}{l}1156.5 \\
1169.8 \\
1211.5 \\
1242.3 \\
1277.6 \\
1317.4 \\
1382.8 \\
1419.6 \\
1456.3 \\
1488.2 \\
1532.2\end{array}$ & $\begin{array}{l}0.6586 \\
0.7112 \\
0.8513 \\
0.9434 \\
1.0641 \\
1.2082 \\
1.6031 \\
2.0151 \\
2.6350 \\
3.5220 \\
8.7910\end{array}$ & $\begin{array}{l}12.536 \\
11.738 \\
10.486 \\
9.9857 \\
9.5542 \\
9.1610 \\
8.5432 \\
8.2125 \\
7.9505 \\
7.7235 \\
7.5090\end{array}$ & $\begin{array}{l}29.892 \\
25.165 \\
17.908 \\
15.123 \\
12.570 \\
10.420 \\
6.8417 \\
4.8633 \\
3.2767 \\
2.1318 \\
0.5499\end{array}$ & $\begin{array}{l}3.0594 \\
3.3854 \\
4.0879 \\
4.4684 \\
4.8944 \\
5.3583 \\
6.4587 \\
7.4299 \\
8.6605 \\
10.189 \\
16.309\end{array}$ & $\begin{array}{l}3.8355 \\
3.9739 \\
4.2868 \\
4.4620 \\
4.6762 \\
4.9087 \\
5.5178 \\
6.1019 \\
6.8855 \\
7.8699 \\
12.247\end{array}$ & $\begin{array}{l}-4.17 \\
-4.17 \\
-4.15 \\
-4.14 \\
-4.12 \\
-4.10 \\
-4.04 \\
-4.00 \\
-3.94 \\
-3.87 \\
-3.64 \\
\end{array}$ & $\begin{array}{l}1.513 \\
1.399 \\
1.226 \\
1.161 \\
1.107 \\
1.058 \\
0.979 \\
0.936 \\
0.903 \\
0.874 \\
0.849\end{array}$ \\
\hline \multicolumn{11}{|c|}{ 313K } \\
\hline $\begin{array}{l}0.000 \\
0.096 \\
0.248 \\
0.308 \\
0.360 \\
0.407 \\
0.479 \\
0.517 \\
0.548 \\
0.574 \\
0.600\end{array}$ & $\begin{array}{l}0.600 \\
0.503 \\
0.352 \\
0.291 \\
0.239 \\
0.192 \\
0.120 \\
0.082 \\
0.051 \\
0.025 \\
0.000\end{array}$ & $\begin{array}{c}860.5 \\
874.4 \\
900.4 \\
912.9 \\
924.5 \\
936.1 \\
957.3 \\
969.3 \\
980.6 \\
991.6 \\
1006.3\end{array}$ & $\begin{array}{l}1132.2 \\
1146.8 \\
1189.6 \\
1216.4 \\
1252.4 \\
1289.2 \\
1353.6 \\
1393.8 \\
1426.6 \\
1462.2 \\
1518.4\end{array}$ & $\begin{array}{l}0.6027 \\
0.6453 \\
0.7566 \\
0.8281 \\
0.9169 \\
1.0320 \\
1.3041 \\
1.6020 \\
2.0330 \\
2.6911 \\
5.7221\end{array}$ & $\begin{array}{l}12.663 \\
11.861 \\
10.612 \\
10.106 \\
9.6751 \\
9.2853 \\
8.6699 \\
8.3506 \\
8.0867 \\
7.8536 \\
7.6023\end{array}$ & $\begin{array}{l}33.076 \\
28.263 \\
20.797 \\
17.817 \\
15.251 \\
12.778 \\
9.0313 \\
6.6743 \\
4.6879 \\
3.1087 \\
1.0330\end{array}$ & $\begin{array}{l}2.9858 \\
3.2867 \\
3.9210 \\
4.2652 \\
4.6245 \\
5.0418 \\
5.9249 \\
6.7193 \\
7.7226 \\
9.0302 \\
13.323\end{array}$ & $\begin{array}{l}3.7811 \\
3.8987 \\
4.1611 \\
4.3104 \\
4.4742 \\
4.6814 \\
5.1368 \\
5.6110 \\
6.2451 \\
7.0920 \\
10.128\end{array}$ & $\begin{array}{l}-4.26 \\
-4.26 \\
-4.25 \\
-4.24 \\
-4.22 \\
-4.20 \\
-4.16 \\
-4.12 \\
-4.06 \\
-4.00 \\
-3.81\end{array}$ & $\begin{array}{l}1.533 \\
1.419 \\
1.248 \\
1.181 \\
1.128 \\
1.079 \\
1.001 \\
0.962 \\
0.928 \\
0.898 \\
0.868\end{array}$ \\
\hline \multicolumn{11}{|c|}{ 318K } \\
\hline $\begin{array}{l}0.000 \\
0.096 \\
0.248 \\
0.308 \\
0.360 \\
0.407 \\
0.479 \\
0.517 \\
0.548 \\
0.574 \\
0.600\end{array}$ & $\begin{array}{l}0.600 \\
0.503 \\
0.352 \\
0.291 \\
0.239 \\
0.192 \\
0.120 \\
0.082 \\
0.051 \\
0.025 \\
0.000\end{array}$ & $\begin{array}{l}851.3 \\
864.9 \\
889.5 \\
901.4 \\
912.5 \\
923.8 \\
943.2 \\
954.9 \\
965.9 \\
977.9 \\
991.2\end{array}$ & $\begin{array}{l}1109.4 \\
1125.8 \\
1170.3 \\
1195.5 \\
1227.6 \\
1264.8 \\
1328.2 \\
1366.9 \\
1402.6 \\
1438.3 \\
1506.3\end{array}$ & $\begin{array}{l}0.5590 \\
0.5980 \\
0.6956 \\
0.7582 \\
0.8331 \\
0.9263 \\
1.1491 \\
1.3790 \\
1.6921 \\
2.2270 \\
4.1951\end{array}$ & $\begin{array}{l}12.800 \\
11.992 \\
10.742 \\
10.235 \\
9.8023 \\
9.4089 \\
8.7995 \\
8.4765 \\
8.2098 \\
7.9636 \\
7.7181\end{array}$ & $\begin{array}{l}35.914 \\
30.813 \\
23.020 \\
19.814 \\
17.089 \\
14.602 \\
10.611 \\
8.1163 \\
6.0191 \\
4.0284 \\
1.6259\end{array}$ & $\begin{array}{l}2.9303 \\
3.2208 \\
3.8193 \\
4.1473 \\
4.4843 \\
4.8564 \\
5.6483 \\
6.3322 \\
7.1464 \\
8.3374 \\
11.519\end{array}$ & $\begin{array}{l}3.7509 \\
3.8625 \\
4.1034 \\
4.2447 \\
4.3956 \\
4.5693 \\
4.9702 \\
5.3675 \\
5.8670 \\
6.6396 \\
8.8910\end{array}$ & $\begin{array}{l}-4.35 \\
-4.35 \\
-4.34 \\
-4.33 \\
-4.31 \\
-4.29 \\
-4.26 \\
-4.22 \\
-4.17 \\
-4.11 \\
-3.95\end{array}$ & $\begin{array}{l}1.556 \\
1.442 \\
1.272 \\
1.205 \\
1.150 \\
1.101 \\
1.025 \\
0.985 \\
0.951 \\
0.919 \\
0.892\end{array}$ \\
\hline
\end{tabular}

The extent of deviation depends upon the nature of the constituents and composition of the mixtures. The thermodynamic excess properties are found to be more sensitive towards intermolecular interaction among the component molecules of liquid mixtures. The sign and extent of deviation of excess parameters depend on the strength of interaction between unlike molecules [22]. 
So various excess acoustic and thermodynamic parameters have been evaluated and corresponding graphs are also given. More over the excess parameters were fitted to Redlich - Kister polynomial and evaluated the coefficients by least square method. These coefficients are reported in the Tables 1.3 and 2.3 followed by the graphs for excess values for the ternary mixtures under study.

The sign and magnitude of excess ultrasonic velocity $\left(\mathrm{U}^{\mathrm{E}}\right)$ play an important role in describing molecular rearrangement as a result of the molecular interactions between the component molecules in the mixtures. The excess ultrasonic velocity $\left(\mathrm{U}^{\mathrm{E}}\right)$ curves at different temperatures varying with mole fraction of monoethylene glycol are represented in the figures, Figure 1.1, and Figure 2.1 for the two ternary systems. The $\mathrm{U}^{\mathrm{E}}$ values starts from negative value reaches negative maximum at around 0.3 mole fraction of MEG and then begins to increase and reaches to positive value at the mole fraction 0.6 of MEG.

Actually the negative trend describes weak interactions among the molecules of the components of the systems. Even with the addition of the third component the same trend is observed, but with the increase in mole fraction of $\mathrm{MEG}$, resultant interactions gradually become stronger, after the mole fraction of 0.3 of MEG. The reason may be stated as, the MEG is self-associated and more polar than the alkyl acetates and the reduction of mole fraction of the IPA/IBA. After the particular mole fraction of MEG, as the concentration of alkyl acetates is decreasing the specific interactions play dominant role than the rupture of hydrogen bond.

\section{Table 1.2. Ternary Liquid Mixture - I : MEG +CY + IPA Excess Values of Acoustic and Thermodynamic Parameters at Temperatures $303,308,313$ and $318 \mathrm{~K}$}

\begin{tabular}{|c|c|c|c|c|c|c|c|c|}
\hline $\begin{array}{c}\text { MEG } \\
\mathrm{X}_{1}\end{array}$ & $\begin{array}{c}\text { IPA } \\
\mathbf{X}_{3}\end{array}$ & $\begin{array}{c}U^{E} \\
\mathrm{~m} / \mathrm{sec}\end{array}$ & $\begin{array}{c}\eta^{\mathrm{E}} \times 10^{-3} \\
\mathrm{Ns} / \mathrm{m}^{2}\end{array}$ & $\begin{array}{l}V_{m}{ }_{m}^{E} \times 10^{-7} \\
m^{3} / \text { mole }\end{array}$ & $\begin{array}{l}V_{f}^{E} \times 10^{-8} \\
m^{3} / \text { mole }\end{array}$ & $\begin{array}{l}\pi_{\mathrm{i}}^{\mathrm{E}} \times 10^{14} \\
\mathrm{~N} / \mathrm{m}^{2}\end{array}$ & $\begin{array}{c}\mathrm{H}^{\mathrm{E}} \times 10^{11} \\
\text { Joule/mol }\end{array}$ & $\begin{array}{c}\Delta \mathrm{G}^{\mathrm{E}} \\
\times \mathbf{1 0}^{10} \\
\text { Joule/mol }\end{array}$ \\
\hline \multicolumn{9}{|c|}{ 303K } \\
\hline 0.0000 & 0.6000 & -72.240 & -0.3596 & 16.419 & 2.2999 & -0.5641 & -0.4988 & -0.6143 \\
\hline 0.1080 & 0.4921 & -91.146 & -0.1476 & 12.473 & 1.3767 & -2.4587 & -1.4011 & -1.1006 \\
\hline 0.1948 & 0.4052 & -102.45 & -0.2331 & 7.4090 & 0.3282 & -3.8604 & -2.0594 & -1.4305 \\
\hline 0.2672 & 0.3330 & -108.15 & -0.3028 & 4.0872 & -0.3265 & -4.9526 & -2.5686 & -1.6529 \\
\hline 0.3798 & 0.2203 & -102.38 & -0.3991 & -0.5311 & -1.1402 & -6.3944 & -3.2021 & -1.8596 \\
\hline 0.4239 & 0.1761 & -93.566 & -0.4281 & -4.1765 & -1.5137 & -6.7676 & -3.3369 & -1.8423 \\
\hline 0.4970 & 0.1032 & -65.156 & -0.4493 & -9.6740 & -2.0894 & -6.9147 & -3.2500 & -1.5482 \\
\hline 0.5272 & 0.0730 & -48.793 & -0.4311 & -13.596 & -2.3805 & -6.5354 & -2.9242 & -1.2324 \\
\hline 0.5536 & 0.0464 & -29.918 & -0.3830 & -16.500 & -2.5619 & -5.7806 & -2.3508 & -0.7990 \\
\hline 0.5782 & 0.0218 & -13.570 & -0.2519 & -19.421 & -2.7450 & -4.0586 & -1.1027 & -0.2979 \\
\hline 0.6001 & 0.0000 & 7.5552 & 0.2484 & -23.229 & -2.8772 & 0.9095 & 2.4283 & 1.6103 \\
\hline \multicolumn{9}{|c|}{ 308K } \\
\hline 0.0000 & 0.6000 & -67.200 & -0.300 & 18.920 & 2.5445 & \multirow{11}{*}{$\begin{array}{c}-0.5517 \\
-2.3280 \\
-3.6555 \\
-4.6835 \\
-6.0594 \\
-6.4528 \\
-6.6500 \\
-6.4102 \\
-5.8872 \\
-4.8090 \\
0.5619\end{array}$} & -0.4820 & -0.6028 \\
\hline 0.1080 & 0.4921 & -85.303 & -1.300 & 15.225 & 1.7331 & & -1.3630 & -1.0947 \\
\hline 0.1948 & 0.4052 & -96.065 & -2.100 & 11.355 & 0.8707 & & -2.0170 & -1.4424 \\
\hline 0.2672 & 0.3330 & -100.50 & -2.700 & 8.8623 & 0.1909 & & -2.5155 & -1.6667 \\
\hline 0.3798 & 0.2203 & -95.927 & -3.600 & 3.8869 & -0.6051 & & -3.1596 & -1.9067 \\
\hline 0.4239 & 0.1761 & -87.956 & -3.900 & 0.5482 & -0.9075 & & -3.3290 & -1.9273 \\
\hline 0.4970 & 0.1032 & -57.395 & -4.100 & -5.6789 & -1.6572 & & -3.3015 & -1.6684 \\
\hline 0.5272 & 0.0730 & -38.676 & -4.000 & -8.5884 & -1.9791 & & -3.0726 & -1.4053 \\
\hline 0.5536 & 0.0464 & -23.570 & -3.700 & -11.566 & -2.2113 & & -2.6620 & -1.0628 \\
\hline 0.5782 & 0.0218 & -8.4460 & -3.000 & -13.118 & -2.4354 & & -1.8489 & -0.4931 \\
\hline 0.6001 & 0.0000 & 6.7169 & 1.860 & -15.438 & -3.0894 & & 2.1337 & 1.5299 \\
\hline \multicolumn{9}{|c|}{ 313K } \\
\hline 0.0000 & 0.6000 & -65.060 & -0.300 & 20.158 & 3.3458 & -0.5558 & -0.4882 & -0.6337 \\
\hline 0.1080 & 0.4921 & -80.825 & -0.900 & 17.428 & 2.7483 & -1.8441 & -1.1150 & -1.0411 \\
\hline 0.1948 & 0.4052 & -91.404 & -1.300 & 13.393 & 1.9260 & -2.7872 & -1.5709 & -1.3289 \\
\hline 0.2672 & 0.3330 & -95.186 & -1.600 & 10.377 & 1.2166 & -3.5061 & -1.9085 & -1.5018 \\
\hline 0.3798 & 0.2203 & -87.129 & -2.100 & 6.2776 & 0.3696 & -4.4373 & -2.3293 & -1.6763 \\
\hline 0.4239 & 0.1761 & -81.475 & -2.200 & 2.4488 & -0.1309 & -4.6756 & -2.4241 & -1.6841 \\
\hline 0.4970 & 0.1032 & -53.366 & -2.300 & -3.0316 & -1.0188 & -4.7011 & -2.3222 & -1.4170 \\
\hline
\end{tabular}




\begin{tabular}{|c|c|c|c|c|c|c|c|c|}
\hline 0.5272 & 0.0730 & -34.000 & -2.200 & -7.1644 & -1.3922 & -4.4854 & -2.1409 & -1.1972 \\
\hline 0.5536 & 0.0464 & -17.507 & -2.000 & -10.140 & -1.7333 & -4.0683 & -1.8224 & -0.9044 \\
\hline 0.5782 & 0.0218 & -3.8710 & -1.400 & -12.120 & -2.2314 & -3.1551 & -1.1380 & -0.3663 \\
\hline 0.6001 & 0.0000 & 6.4378 & 1.340 & -16.430 & -3.3037 & 0.6307 & 1.6649 & 1.3184 \\
\hline \multicolumn{9}{|c|}{ 318K } \\
\hline 0.0000 & 0.6000 & -62.960 & \multirow{11}{*}{$\begin{array}{l}-0.299 \\
-0.702 \\
-0.900 \\
-1.201 \\
-1.401 \\
-1.506 \\
-1.500 \\
-1.400 \\
-1.200 \\
-0.800 \\
0.950\end{array}$} & 22.515 & \multirow{11}{*}{$\begin{array}{c}3.7816 \\
3.1392 \\
2.5009 \\
1.8805 \\
1.0560 \\
0.5963 \\
-0.4405 \\
-0.8767 \\
-1.4312 \\
-1.9524 \\
-3.3469\end{array}$} & -0.5418 & -0.4692 & \\
\hline 0.1080 & 0.4921 & -77.522 & & 19.254 & & -1.5439 & -0.9431 & $\begin{array}{r}-0.6244 \\
0.0530\end{array}$ \\
\hline 0.1948 & 0.4052 & -88.266 & & 14.786 & & -2.2727 & -1.2912 & -0.9538 \\
\hline 0.2672 & 0.3330 & -91.148 & & 11.883 & & -2.8202 & -1.5420 & -1.2010 \\
\hline 0.3798 & 0.2203 & -82.543 & & 7.7990 & & -3.5066 & -1.8367 & $\begin{array}{r}-1.3385 \\
1.1632\end{array}$ \\
\hline 0.4239 & 0.1761 & -76.780 & & 4.6023 & & -3.6540 & -1.8861 & -1.4632 \\
\hline 0.4970 & 0.1032 & -49.479 & & -1.5370 & & -3.6078 & -1.7687 & -1.4529 \\
\hline 0.5272 & 0.0730 & -28.348 & & -5.1663 & & -3.4058 & -1.6050 & -1.2045 \\
\hline 0.5536 & 0.0464 & -12.352 & & -8.1243 & & -2.9887 & -1.2890 & -0.9989 \\
\hline 0.5782 & 0.0218 & -3.5890 & & -10.461 & & -2.2963 & -0.7761 & -0.6986 \\
\hline 0.6001 & 0.0000 & 6.3786 & & -12.517 & & 0.4699 & 1.3120 & $\begin{array}{r}-0.2737 \\
1.1422\end{array}$ \\
\hline
\end{tabular}

Table 1.3. Values of the Coefficients of Redlich - Kister Polynomial and Standard Deviation $(\sigma)$ for Ternary Mixture - I

\begin{tabular}{|c|c|c|c|c|c|}
\hline Temp. , K & $\mathbf{A}_{0}$ & $\mathbf{A}_{1}$ & $\mathbf{A}_{2}$ & $\mathbf{A}_{3}$ & $\boldsymbol{\sigma}$ \\
\hline \multicolumn{6}{|c|}{ Excess Ultrasonic velocity $\mathbf{U}^{\mathrm{E}} \mathrm{m} / \mathrm{sec}$} \\
\hline 303 & -3599.51 & 24882.8 & -274883.8 & 832898.4 & 7.9011 \\
\hline 308 & -3365.88 & 23029.2 & -263196.9 & 889103.2 & 7.6231 \\
\hline 313 & -3179.94 & 26803.2 & -332720.9 & 1196557.4 & 6.1202 \\
\hline 318 & -3060.55 & 22366.8 & -254834.1 & 938043.6 & 6.4454 \\
\hline \multicolumn{6}{|c|}{ Excess Viscosity $\eta^{\mathrm{E}} \times \mathbf{1 0}^{-3} \mathrm{Ns} / \mathrm{m}^{2}$} \\
\hline 303 & -7.6046 & -103.152 & 1016.77 & -6507.35 & 0.0144 \\
\hline 308 & -69.677 & -1871.62 & 27286.6 & -133829.4 & 0.4609 \\
\hline 313 & -43.536 & -582.991 & 7450.47 & -43809.3 & 0.1481 \\
\hline 318 & -31.932 & -246.593 & 2611.23 & -18668.5 & 0.0788 \\
\hline \multicolumn{6}{|c|}{$\mathrm{V}_{\mathrm{m}}^{\mathrm{E}} \times 10^{-7} \mathrm{~m}^{3} / \mathrm{mole}$} \\
\hline 303 & 312.264 & -24402.3 & 367117 & -1545944 & 4.4013 \\
\hline 308 & 452.631 & -19086.8 & 261716 & -1078210 & 3.0017 \\
\hline 313 & 527.816 & -19700.3 & 267295 & -1080540 & 2.9890 \\
\hline 318 & 519.962 & -21681.5 & 293053 & -1128860 & 4.0830 \\
\hline \multicolumn{6}{|c|}{$\mathrm{V}_{\mathrm{f}}^{\mathrm{E}} \times 10^{-7} \mathrm{~m}^{3} / \mathrm{mole}$} \\
\hline 303 & 22.8699 & -3487.99 & 49523.4 & -205072 & 0.5777 \\
\hline 308 & 39.5962 & -3324.12 & 46252.6 & -189604 & 0.5042 \\
\hline 313 & 77.5122 & -3780.41 & 50806.1 & -200452 & 0.5889 \\
\hline 318 & 96.0603 & -3626.21 & 48680.6 & -190893 & 0.5394 \\
\hline \multicolumn{6}{|c|}{$\pi_{\mathrm{i}}^{\mathrm{E}} \times 10^{15} \mathrm{~N} / \mathrm{m}^{2}$} \\
\hline 303 & -126.466 & -2001.88 & 23649.0 & -129703.8 & 0.3749 \\
\hline 308 & -122.109 & -30229.5 & 44740.7 & -217327.8 & 0.7630 \\
\hline 313 & -93.1224 & -1795.98 & 25768.6 & -129299.2 & 0.4527 \\
\hline 318 & -76.0328 & -1238.08 & 17445.1 & -88712.8 & 0.3279 \\
\hline \multicolumn{6}{|c|}{$\mathbf{H}^{\mathrm{E}} \times 10^{11}$ Joule/mole } \\
\hline 303 & -66.4654 & 69.3146 & -7940.10 & 19420.0 & 0.1493 \\
\hline 308 & -66.7816 & -821.644 & 9561.68 & -54507.7 & 0.1873 \\
\hline 313 & -52.1960 & -320.838 & 2527.21 & -21311.0 & 0.0868 \\
\hline 318 & -42.9999 & -134.041 & 137.474 & -8340.58 & 0.0559 \\
\hline \multicolumn{6}{|c|}{$\Delta G^{\mathrm{E}} \times 10^{10}$ Joule/mole } \\
\hline 303 & -47.6747 & 250.113 & -6565.54 & 23497.4 & 0.0625 \\
\hline 308 & -48.0224 & 139.160 & -4445.23 & 12420.2 & 0.0603 \\
\hline 313 & -44.5569 & 249.735 & -5562.25 & 17162.0 & 0.0771 \\
\hline 318 & -40.3550 & 259.691 & -5319.47 & 17337.0 & 0.0613 \\
\hline
\end{tabular}



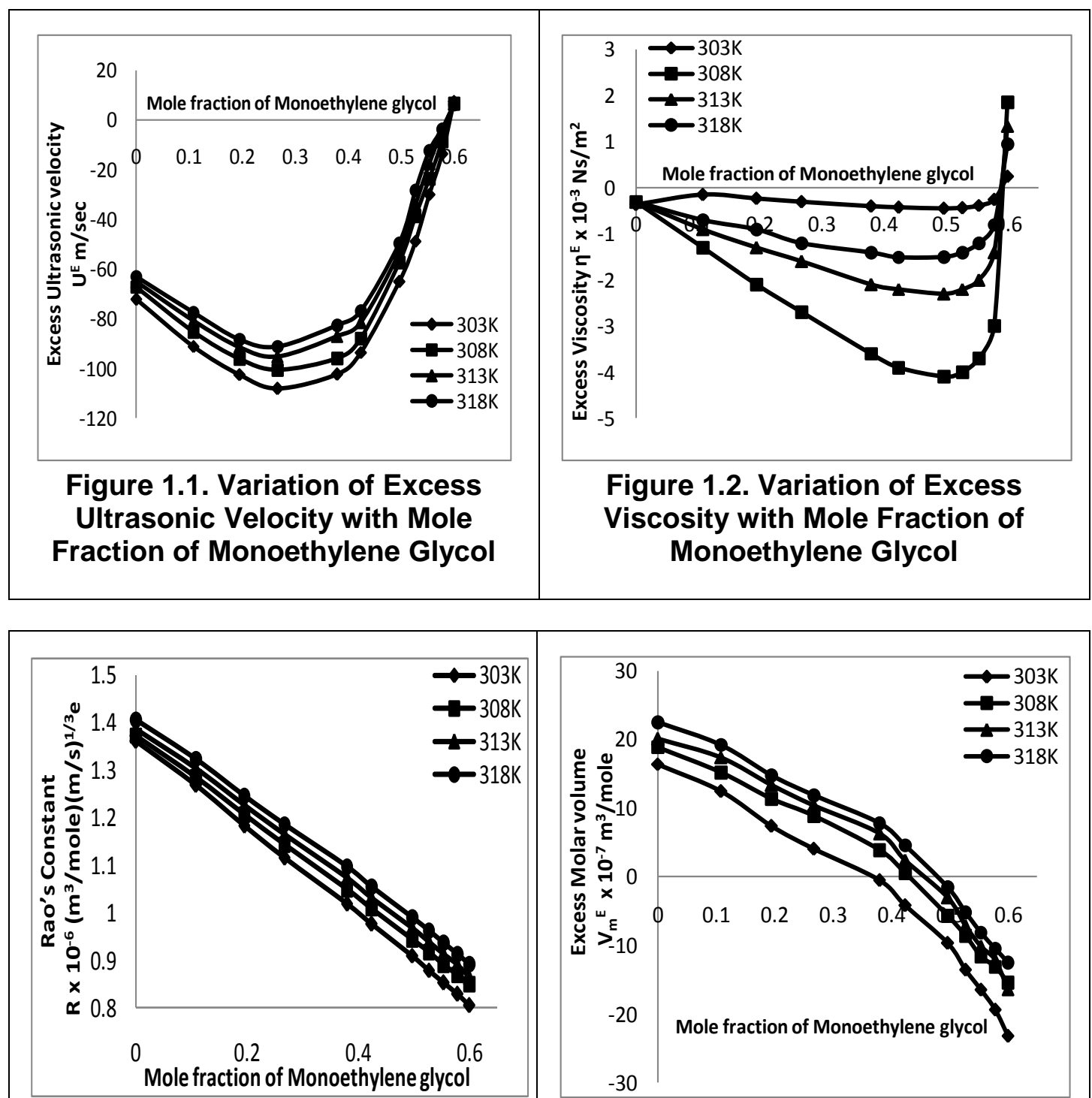

Figure 1.3. Variation of Rao's Constant with Mole Fraction of Monoethylene Glycol

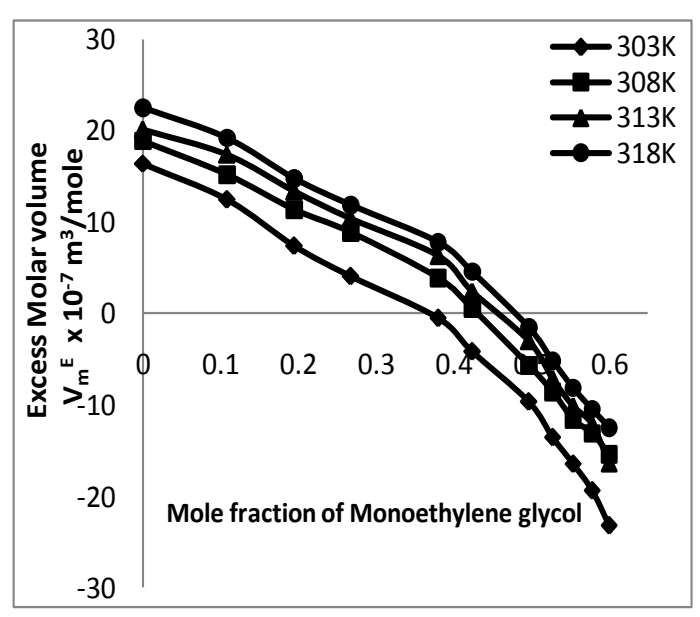

Figure 1.4. Variation of Excess Molar Volume with Mole Fraction of Monoethylene Glycol

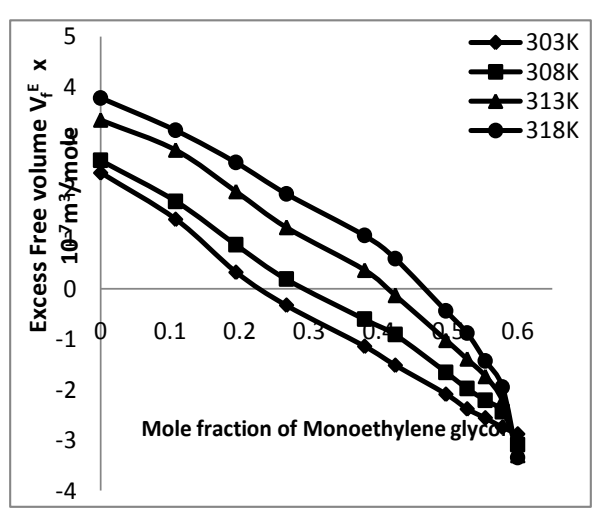

Figure 1.5. Variation of Excess Free Volume with Mole Fraction of Monoethylene Glycol

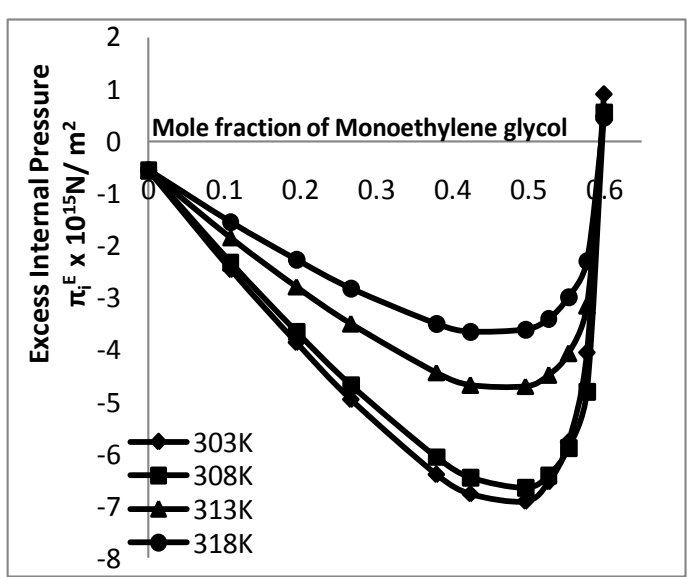

Figure 1.6. Variation of Excess Internal Pressure with Mole Fraction of Monoethylene Glycol 


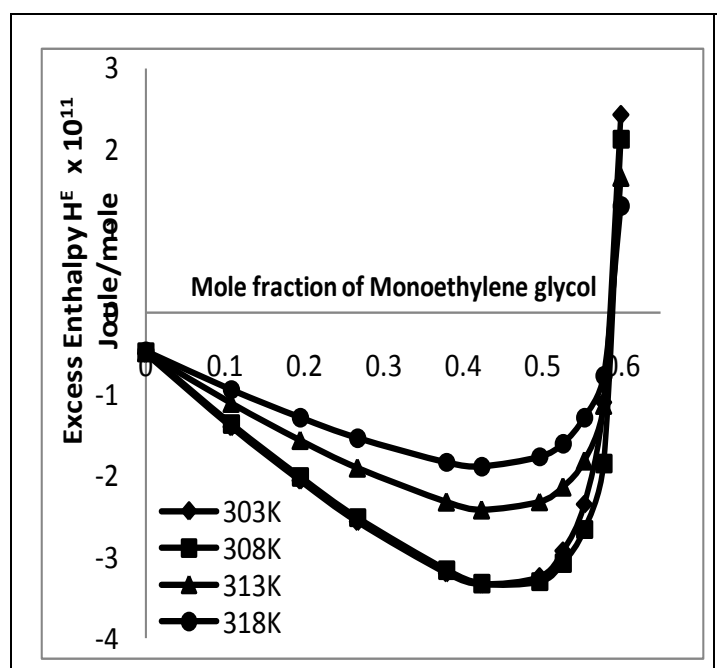

Figure 1.7. Variation of Excess Enthalpy with Mole Fraction of Monoethylene Glycol

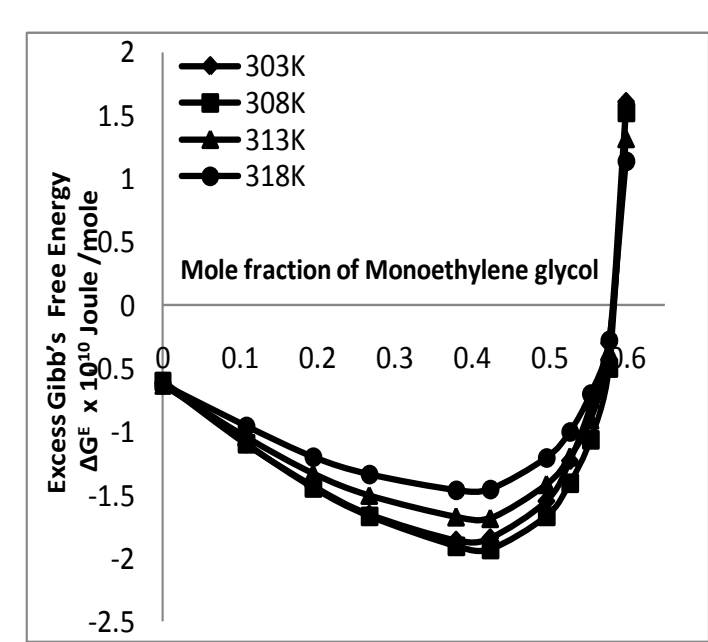

Figure 1.8. Variation of Excess Gibb's Free Energy with Mole Fraction of Monoethylene Glycol

Table 2.2. Ternary Liquid Mixture - II : MEG +CY + IBA Excess Values of Acoustic and Thermodynamic Parameters at Temperatures $303,308,313$ and $318 \mathrm{~K}$

\begin{tabular}{|c|c|c|c|c|c|c|c|c|}
\hline $\begin{array}{c}\text { Mole } \\
\text { fraction } \\
\text { of } M E G \\
X_{1} \\
\end{array}$ & $\begin{array}{c}\text { Mole } \\
\text { fraction } \\
\text { of IBA } \\
\mathbf{X}_{\mathbf{3}} \\
\end{array}$ & $\begin{array}{c}\mathbf{U}^{\mathrm{E}} \\
\mathbf{m} / \mathbf{s e c}\end{array}$ & $\begin{array}{c}\eta^{\mathrm{E}} \times 10^{-3} \\
\mathrm{Ns} / \mathrm{m}^{2}\end{array}$ & $\begin{array}{l}V_{m}^{E} \times 10^{-7} \\
m^{3} / \text { mole }\end{array}$ & $\begin{array}{l}V_{f}^{E} \times 10^{-8} \\
m^{3} / \text { mole }\end{array}$ & $\begin{array}{l}\pi_{i}^{\mathrm{E}} \times 10^{14} \\
\mathrm{~N} / \mathrm{m}^{2}\end{array}$ & $\begin{array}{c}\mathrm{H}^{\mathrm{E}} \times 10^{11} \\
\text { Joule/mol }\end{array}$ & $\begin{array}{c}\Delta G^{\mathrm{E}} \\
\mathrm{x} \mathbf{1 0}^{10} \\
\text { Joule/mol }\end{array}$ \\
\hline \multicolumn{9}{|c|}{ 303K } \\
\hline 0.0000 & 0.6001 & -48.593 & -0.3541 & 18.690 & 3.4347 & -0.6477 & -0.5421 & -0.6113 \\
\hline 0.0968 & 0.5032 & -86.938 & -1.3658 & 13.789 & 2.3967 & -2.3711 & -1.3498 & -1.0935 \\
\hline 0.2480 & 0.3521 & -119.71 & -2.8772 & 5.2153 & 0.8798 & -4.8587 & -2.5127 & -1.6973 \\
\hline 0.3087 & 0.2913 & -118.94 & -3.4348 & 1.1653 & 0.2211 & -5.7295 & -2.9100 & -1.8594 \\
\hline 0.3609 & 0.2392 & -106.13 & -3.8623 & -2.8232 & -0.4382 & -6.3683 & -3.1850 & -1.9089 \\
\hline 0.4074 & 0.1926 & -89.876 & -4.1857 & -5.8582 & -1.0171 & -6.8134 & -3.3531 & -1.8920 \\
\hline 0.4797 & 0.1202 & -59.452 & -4.4539 & -10.782 & -1.9379 & -7.0502 & -3.3209 & -1.6360 \\
\hline 0.5179 & 0.0821 & -41.362 & -4.3148 & -13.118 & -2.3844 & -6.7103 & -2.9922 & -1.282 \\
\hline 0.5483 & 0.0517 & -27.757 & -3.8765 & -16.168 & -2.6032 & -5.9643 & -2.4260 & -0.8416 \\
\hline 0.5748 & 0.0253 & -11.665 & -2.7842 & -19.554 & -2.7261 & -4.4584 & -1.3567 & -0.1606 \\
\hline 0.6000 & 0.0000 & 7.7200 & 2.4856 & -23.232 & -2.8772 & 0.9136 & 2.4299 & 1.6065 \\
\hline \multicolumn{9}{|c|}{$308 \mathrm{~K}$} \\
\hline 0.0000 & 0.6001 & -52.910 & -0.3 & 21.157 & 4.4226 & -0.6601 & -0.5594 & -0.6639 \\
\hline 0.0968 & 0.5032 & -90.485 & -1.2 & 16.658 & 3.2206 & -2.2740 & -1.3430 & -1.1450 \\
\hline 0.2480 & 0.3521 & -128.53 & -2.6 & 8.7773 & 1.4604 & -4.6019 & -2.4712 & -1.7582 \\
\hline 0.3087 & 0.2913 & -129.59 & -3.1 & 5.9808 & 0.8866 & -5.4377 & -2.8741 & -1.9414 \\
\hline 0.3609 & 0.2392 & -121.90 & -3.5 & 3.2340 & 0.2295 & -6.0581 & -3.1576 & -2.0147 \\
\hline 0.4074 & 0.1926 & -106.48 & -3.8 & 0.1665 & -0.2255 & -6.5259 & -3.3678 & -2.0436 \\
\hline 0.4797 & 0.1202 & -79.048 & -4.1 & -5.3221 & -1.1703 & -6.8742 & -3.4473 & -1.8796 \\
\hline 0.5179 & 0.0821 & -62.478 & -4.1 & -8.8565 & -1.7627 & -6.6688 & -3.2276 & -1.5899 \\
\hline 0.5483 & 0.0517 & -41.790 & -3.8 & -11.447 & -2.2435 & -6.0475 & -2.7337 & -1.1443 \\
\hline 0.5748 & 0.0253 & -23.958 & -3.2 & -13.696 & -2.4280 & -5.0498 & -2.0021 & -0.6092 \\
\hline 0.6000 & 0.0000 & 6.8800 & 1.86 & -15.441 & -3.0894 & 0.5657 & 2.1352 & 1.5260 \\
\hline \multicolumn{9}{|c|}{ 313K } \\
\hline 0.0000 & 0.6001 & -58.829 & -0.3 & 22.895 & 5.0564 & -0.6386 & -0.5364 & -0.6780 \\
\hline 0.0968 & 0.5032 & -95.889 & -0.8 & 18.416 & 4.0675 & -1.8026 & -1.0862 & -1.0828 \\
\hline 0.2480 & 0.3521 & -134.06 & -1.6 & 11.296 & 2.5648 & -3.4576 & -1.8671 & -1.5915 \\
\hline 0.3087 & 0.2913 & -139.61 & -1.9 & 8.1706 & 1.9841 & -4.0321 & -2.1361 & -1.7438 \\
\hline 0.3609 & 0.2392 & -131.64 & -2.1 & 5.6661 & 1.4746 & -4.4633 & -2.3327 & -1.8079 \\
\hline 0.4074 & 0.1926 & -119.60 & -2.2 & 3.1022 & 0.8406 & -4.7497 & -2.4459 & -1.8074 \\
\hline 0.4797 & 0.1202 & -93.755 & -2.4 & -1.8851 & -0.0490 & -4.9608 & -2.4888 & -1.6859 \\
\hline 0.5179 & 0.0821 & -74.093 & -2.3 & -4.1478 & -0.9024 & -4.7450 & -2.2785 & -1.4045 \\
\hline 0.5483 & 0.0517 & -57.551 & -2.1 & -6.8203 & -1.6891 & -4.2018 & -1.8541 & -0.9959 \\
\hline
\end{tabular}




\begin{tabular}{|l|l|l|l|l|l|l|l|l|}
\hline 0.5748 & 0.0253 & -36.232 & -1.5 & -9.5902 & -2.2265 & -3.2957 & -1.1904 & -0.4494 \\
0.6000 & 0.0000 & 6.6000 & 1.34 & -14.924 & -3.3037 & 0.6161 & 1.6728 & 1.3195 \\
\hline \multicolumn{7}{|c|}{$\mathbf{3 1 8 K}$} \\
\hline 0.0000 & 0.6001 & -63.707 & -0.3 & 24.729 & 5.2850 & -0.6267 & -0.5201 & -0.6866 \\
0.0968 & 0.5032 & -99.917 & -0.6 & 20.027 & 4.3272 & -1.5444 & -0.9417 & -1.0342 \\
0.2480 & 0.3521 & -137.87 & -1.1 & 13.554 & 2.9935 & -2.8332 & -1.5346 & -1.4665 \\
0.3087 & 0.2913 & -145.62 & -1.3 & 10.589 & 2.3878 & -3.2634 & -1.7275 & -1.5899 \\
0.3609 & 0.2392 & -142.05 & -1.5 & 8.1465 & 1.8893 & -3.5783 & -1.8647 & -1.6388 \\
0.4074 & 0.1926 & -130.07 & -1.6 & 5.4305 & 1.3953 & -3.7864 & -1.9469 & -1.6388 \\
0.4797 & 0.1202 & -105.94 & -1.6 & 1.3647 & 0.5000 & -3.8968 & -1.9443 & -1.5109 \\
0.5179 & 0.0821 & -88.148 & -1.5 & -1.0974 & -0.3666 & -3.6901 & -1.7579 & -1.2608 \\
0.5483 & 0.0517 & -69.004 & -1.3 & -3.9201 & -1.1642 & -3.2554 & -1.4259 & -0.9139 \\
0.5748 & 0.0253 & -47.843 & -0.9 & -7.8782 & -2.0262 & -2.3954 & -0.7998 & -0.3589 \\
0.6000 & 0.0000 & 6.5400 & 0.95 & -12.520 & -3.3469 & 0.4725 & 1.3130 & 1.1379 \\
\hline
\end{tabular}

Table 1.3. Values of the Coefficients of Redlich - Kister Polynomial and Standard Deviation ( $\sigma$ ) for Ternary Mixture - II

\begin{tabular}{|c|c|c|c|c|c|}
\hline Temp, K & $\mathbf{A}_{\mathbf{0}}$ & $\mathbf{A}_{1}$ & $\mathbf{A}_{2}$ & $\mathbf{A _ { 3 }}$ & $\boldsymbol{\sigma}$ \\
\hline \multicolumn{6}{|c|}{ Excess Ultrasonic velocity $\mathrm{U}^{\mathrm{E}} \mathrm{m} / \mathrm{sec}$} \\
\hline 303 & -4459.68 & 18773.2 & --95614.2 & -124037.8 & 7.7175 \\
\hline 308 & -4222.06 & 22720.2 & -167286.9 & 306955.1 & 5.4511 \\
\hline 313 & -4459.68 & 18773.2 & -95614.2 & -124037.8 & 7.7175 \\
\hline 318 & -4627.17 & 14485.9 & -26755.3 & -521828.2 & 10.104 \\
\hline \multicolumn{6}{|c|}{ Excess Viscosity $\eta^{\mathrm{E}} \times 10^{-3} \mathrm{Ns} / \mathrm{m}^{2}$} \\
\hline 303 & -76.2102 & -986.691 & 9871.98 & -64190.1 & 0.1680 \\
\hline 308 & -68.0309 & -1436.02 & 20315.3 & -109808.0 & 0.3742 \\
\hline 313 & -43.6504 & -465.430 & 4931.43 & -33992.6 & 0.0867 \\
\hline 318 & -31.2847 & -272.830 & 2255.65 & -16727.2 & 0.0771 \\
\hline \multicolumn{6}{|c|}{$\mathrm{V}_{\mathrm{m}}^{\mathrm{E}} \times 10^{-7} \mathrm{~m}^{3} / \mathrm{mole}$} \\
\hline 303 & 450.625 & -25198.1 & 331014 & -1357711 & 3.6607 \\
\hline 308 & 581.072 & -21412.0 & 259632 & -1028359 & 2.8546 \\
\hline 313 & 662.521 & -21044.1 & 242845 & -897992.6 & 2.8208 \\
\hline 318 & 737.518 & -21700.6 & 252009 & -903904.1 & 2.9101 \\
\hline \multicolumn{6}{|c|}{$\mathrm{V}_{\mathrm{f}}^{\mathrm{E}} \times 10^{-7} \mathrm{~m}^{3} / \mathrm{mole}$} \\
\hline 303 & 78.4723 & -3690.46 & 42909.3 & -172698 & 0.4534 \\
\hline 308 & 109.179 & -4141.61 & 47716.1 & -183860 & 0.5186 \\
\hline 313 & 147.001 & -4559.57 & 54453.1 & -206358 & 0.5725 \\
\hline 318 & 159.594 & -4725.74 & 57748.0 & -215470 & 0.5879 \\
\hline \multicolumn{6}{|c|}{$\pi_{\mathrm{i}}^{\mathrm{E}} \times 10^{15} \mathrm{~N} / \mathrm{m}^{2}$} \\
\hline 303 & -130.409 & -1711.36 & 19427.6 & -115352 & 0.3696 \\
\hline 308 & -124.427 & -2172.48 & 30468.7 & -166056 & 0.6248 \\
\hline 313 & -95.9974 & -1187.57 & 15333.8 & -91071.7 & 0.3462 \\
\hline 318 & -80.5264 & -685.852 & 8010.22 & -54713.2 & 0.2089 \\
\hline \multicolumn{6}{|c|}{$\mathrm{H}^{\mathrm{E}} \times 10^{11}$ Joule/mole } \\
\hline 303 & -70.6459 & -156.481 & -3470.98 & 2883.83 & 0.0606 \\
\hline 308 & -70.0072 & -587.030 & 5949.06 & -41595.5 & 0.1771 \\
\hline 313 & -54.8128 & -135.360 & -707.311 & -8605.36 & 0.0635 \\
\hline 318 & -46.4136 & 76.1483 & -3460.36 & 5154.56 & 0.0489 \\
\hline \multicolumn{6}{|c|}{$\Delta G^{\mathrm{E}} \times 10^{10}$ Joule/mole } \\
\hline 303 & -52.6987 & 418.875 & -9426.03 & 36577.2 & 0.1253 \\
\hline 308 & -54.9616 & 181.581 & -4486.72 & 11940.2 & 0.0554 \\
\hline 313 & -51.0470 & 311.461 & -6369.21 & 20052.9 & 0.0645 \\
\hline 318 & -48.0843 & 386.898 & -7202.90 & 23406.8 & 0.0800 \\
\hline
\end{tabular}




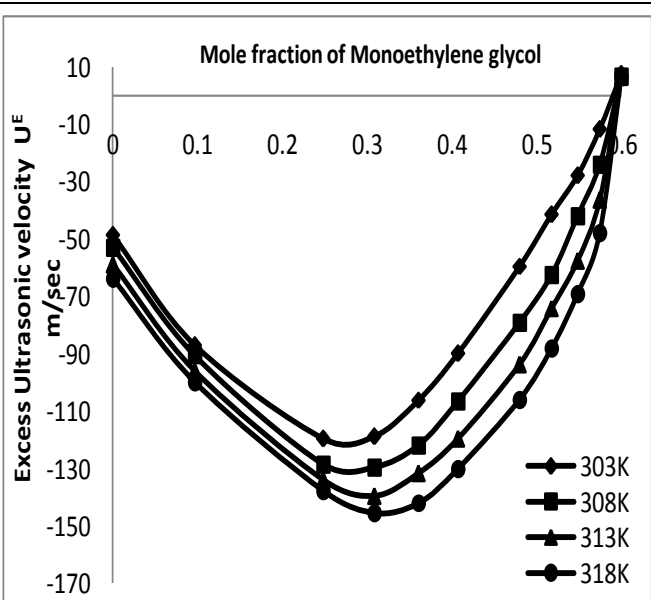

Figure 2.1. Variation of Excess Ultrasonic Velocity with Mole Fraction of Monoethylene Glycol

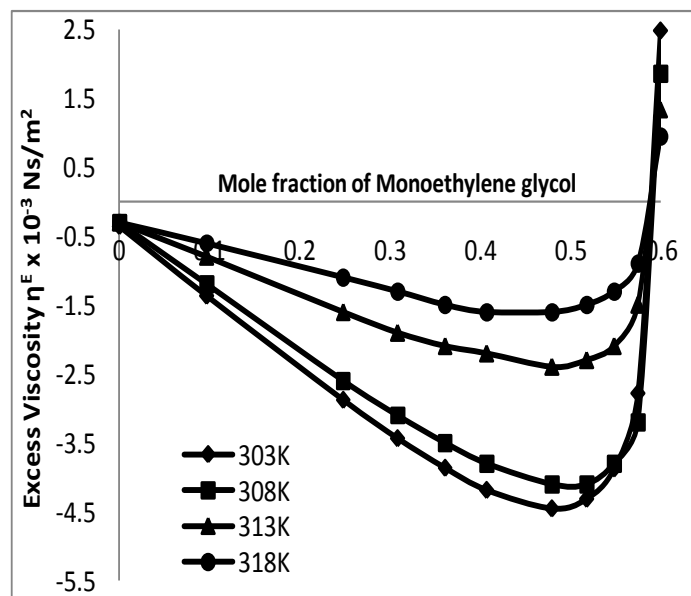

Figure 2.2. Variation of Excess Viscosity with Mole Fraction of Monoethylene Glycol

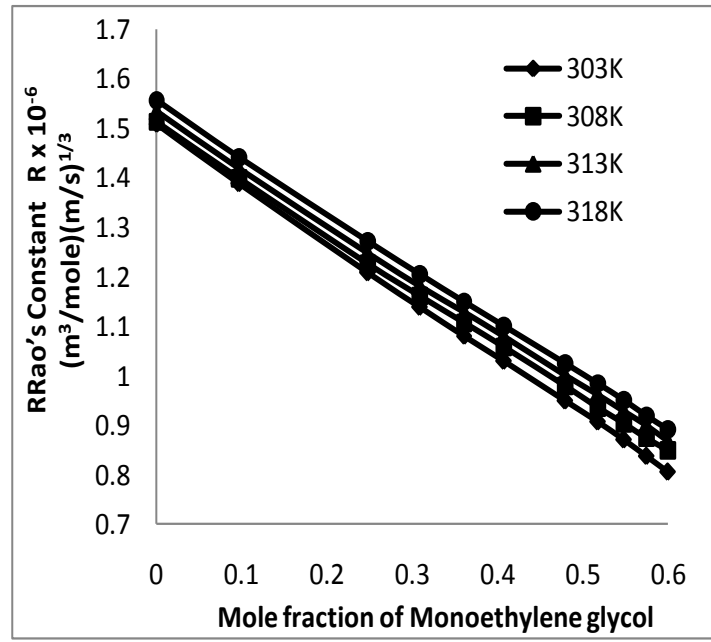

Figure 2.3. Variation of Rao's Constant with Mole Fraction of Monoethylene Glycol

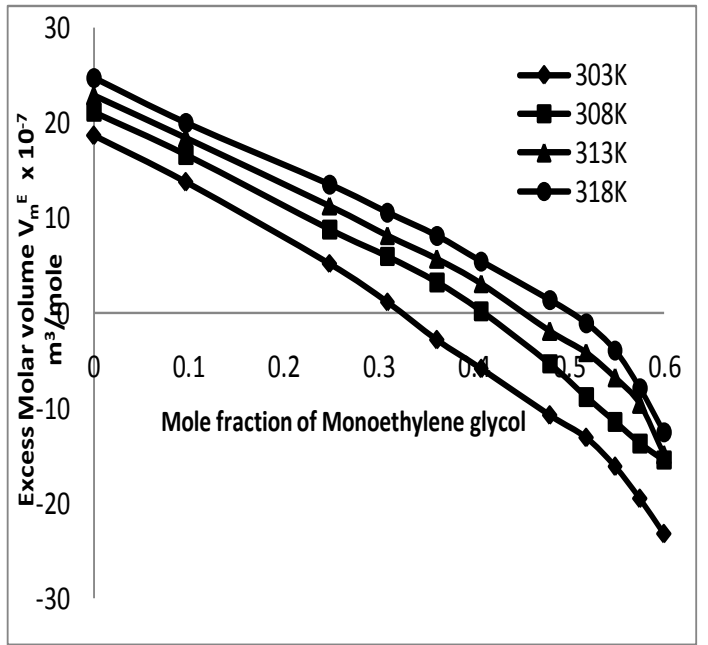

Figure 2.4. Variation of Excess Molar Volume with Mole Fraction of Monoethylene Glycol

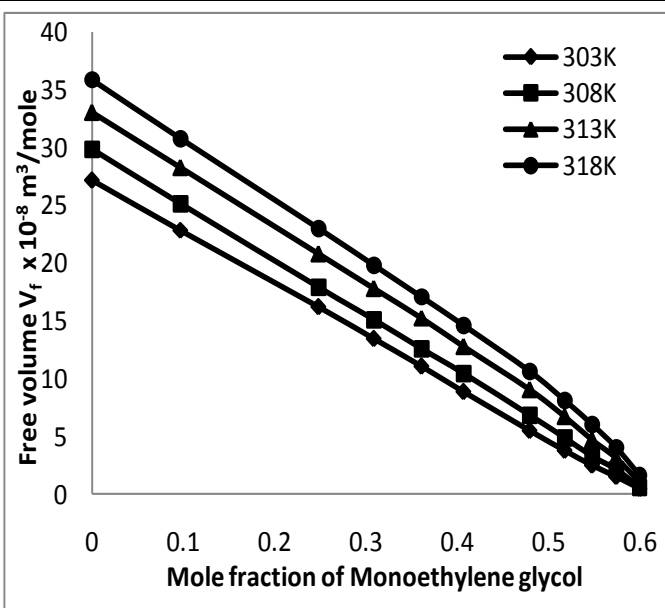

Figure 2.5. Variation of Excess Free Volume with Mole Fraction of Monoethylene Glycol

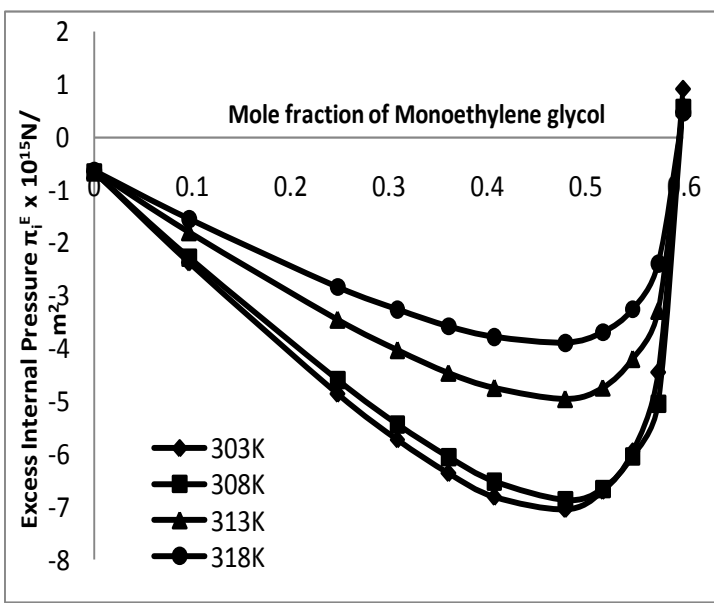

Figure 2.6. Variation of Excess Internal Pressure with Mole Fraction of Monoethylene Glycol 


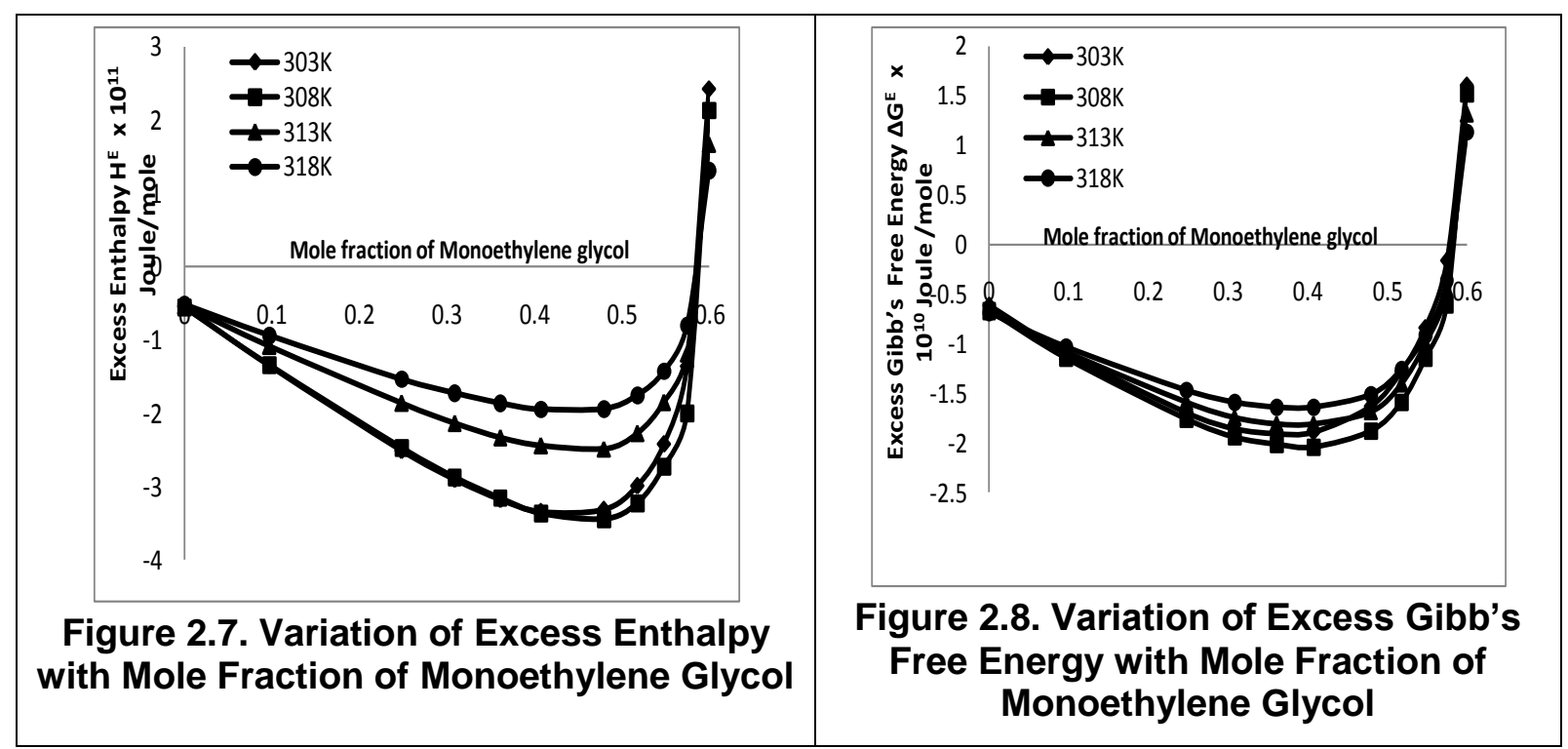

The measurement of viscosity in ternary liquid mixture gives some reliable information in the study of intermolecular interaction. The molecules of one or more components forming the ternaries are either polar, associating and accordingly show non-ideal behaviors in mixtures. Negative values of $\eta^{\mathrm{E}}$ in most of the cases are the consequence of lower viscosity contributions of similar non-specific interactions and H-bonding effects of molecular species in real mixtures rather than those in the corresponding ideal mixtures.

In the present study, it is observed that, for the two ternary systems the $\eta^{\mathrm{E}}$ values gradually decrease up to the mole fraction around 0.5 and then begins to increase (can be seen form Figure 1.2 and Figure 2.2. More over it is observed that the $\eta^{\mathrm{E}}$ values decrease as the temperature increases. The negative values imply the presence of dispersion forces between the mixing components in the mixtures, and the positive values may be attributed to the presence of specific interactions.

The excess molar volume $\left(\mathrm{V}_{\mathrm{m}}^{\mathrm{E}}\right)$ is another important parameter through which molecular interactions can be explained. In the present investigation, for the ternary systems it is observed that, as the mole fraction of MEG increases, the $\mathrm{V}_{\mathrm{m}}^{\mathrm{E}}$ values continuously decreasing (begins with positive, ends with negative values) (Figure 1.4, Figure 2.4). So this kind of trend may be interpreted as, the interactions among the molecules of the component mixtures, are moving from weak to strong. As the temperature is increasing, the $\mathrm{V}_{\mathrm{m}}{ }^{\mathrm{E}}$ values are decreasing in all the four ternary systems.

These effects may be arbitrarily divided into three types, namely chemical, physical and structural. A physical contribution, that is from non-specific interactions between the real species present in the mixture, contributes a positive trend to $\mathrm{V}_{\mathrm{m}} \mathrm{E}$. The chemical or specific intermolecular interactions result in a volume decrease, and these interactions include charge transfer type forces and other complex-forming interactions. This effect yields negative contributions to $\mathrm{V}_{\mathrm{m}}^{\mathrm{E}}$. The structural contributions are mostly negative and arise from several effects, especially from interstitial accommodation changes the free volume. In other words, structural contributions arising from geometrical fitting of one component into the other because of differences in the free volume and molar volume between components lead to a overall negative contribution to $\mathrm{V}_{\mathrm{m}}{ }^{\mathrm{E}}$. Here in the present mixtures, it may be concluded that the chemical or specific intermolecular interactions dominate over the other types of interactions [23, 24].

In the ternary mixtures, the excess free volume $\left(\mathrm{V}_{\mathrm{f}}^{\mathrm{E}}\right)$ values decreases non-linearly beginning from positive to the negative as the mole fraction of MEG increases, which can be seen from the plots Figure 1.5, Figure 2.5. Adgaonkar et. al., and others [26] showed positive values of $\mathrm{V}_{\mathrm{f}}^{\mathrm{E}}$ indicate the existence of weak molecular interactions in the liquid mixtures and the negative values of excess free volume suggest the specific interactions among unlike molecules. The negative values of excess free volume $\left(V_{f}^{E}\right)$ in the systems 
assert that the combined effect of the factors responsible for volume contraction and viceversa. More over, the interactions include charge transfer type forces and other complexforming interactions resulting specific interactions dominate over the other forces acting among the molecules.

A qualitative explanation of the behaviour of the ternary mixtures has been suggested as follows: The mixing of $\mathrm{CY}$ with glycols causes the dissociation of the hydrogenbonded structure of glycols and the subsequent formation of (new) $\mathrm{H}$-bonds $[\mathrm{C}=\mathrm{O}$......H$\mathrm{O}$ ] between the proton acceptor oxygen atom (with lone pair of electrons) of the $\mathrm{C}=\mathrm{O}$ group of $\mathrm{CY}$ and the proton of the $\mathrm{OH}$ group of glycol. The first (dissociation) effect leads to an increase in free volume, resulting in positive values, whereas the second effect leads to a reduction in free volume, resulting in negative values of $\mathrm{V}_{\mathrm{f}}^{\mathrm{E}}$. So here it is observed that the interactions are moving form weak to strong as the concentration of MEG increases.

It is an interesting fact to note that the variation of excess internal pressure, excess enthalpy and excess Gibb's free energy exhibit the same trend as that of the excess viscosity and behave exactly in a reverse trend as that of the free volume at all the four temperatures.

The values of $\pi_{\mathrm{i}}^{\mathrm{E}}$ are almost negative and gradually decrease and move towards the positive values by the increase of mole fraction of MEG. More over the $\pi_{\mathrm{i}}^{\mathrm{E}}$ decreases with increase in temperature. This situation is observed for all the four ternary systems under study and can be viewed from plots, Figure 1.6, Figure 2.6. This suggests that dipole and dispersive forces are operative in these systems, when the MEG concentration is low. When the concentration of MEG increased, the corresponding decrease in concentration of alkyl acetates leads to specific interactions; i.e., the interactions move from weak to strong which supports the above arguments in case of other parameters.

The variation of excess enthalpy $\left(\mathrm{H}^{\mathrm{E}}\right)$ with the mole fraction of MEG can be observed in the plots, Figure 1.7, Figure 2.7 for all the four mixtures. The $\mathrm{H}^{\mathrm{E}}$ values decrease with increase in temperature is observed in the present study. The gradual increase from negative to positive values of $\mathrm{H}^{\mathrm{E}}$, indicates that interactions are moving from exothermic to endothermic effect. As the concentration of MEG increases the endothermic effect dominates the exothermic effect leads to strong interactions among the components of the mixture.

Next, the excess Gibb's free energy $\left(\Delta \mathrm{G}^{\mathrm{E}}\right)$ is negative at low concentration of MEG in the mixtures and positive in the MEG rich concentration which can be seen from the figures, Figure 1.8 and Figure 2.8. The increase of $\Delta \mathrm{G}^{\mathrm{E}}$ values with increase in temperature is also observed. In the negative region the trend is due to the rupture of hydrogen bond and dominance of dispersive forces. Where as the positive trend suggest the complex formation among the components of the mixture indicating strong interactions. So the present argument is complimented by the above discussion. The reduction of negative excess values with increase in concentration of MEG, in all the four ternary systems indicates the need for smaller time for the cooperative process; or the rearrangement of molecules in mixtures decreases energy that leads to dissociation. Recently Ali and Nain[26] have attributed the increasing positive values of Gibb's free energy in few ternary liquid mixtures to hydrogen bond formation between the unlike molecules, which supports the present investigation.

\section{Conclusion}

The above discussion reveal that the existence of molecular interactions in the ternary liquid mixtures. The present investigation shows that greater molecular interaction exists in the mixtures which may be due to hydrogen bonding formation. Also the weak molecular interaction that exists which may be due to the dominance of dispersion forces and dipolar interaction between the unlike molecules. In this manner all the parameters which have been evaluated form the measured values support one another to give a conclusion that the interactions becoming strong starting from weak interactions among the component molecules of the mixtures. 


\section{Acknowledgments}

One of the authors P. Paul Divakar highly thankful to UGC, India for awarding FIP under XI- Plan to do Ph.D in Andhra University during the period 2009-2011, and also thankful to Prof. K.Samatha, the research director.

\section{References}

[1] K. T. Jacob and K. Fitzner, Thermochim. Acta., vol. 18, (1977), pp. 197-206.

[2] R. P. Rastogi, J. Nath and S. S. Das, Journal of Chem. Eng. Data, vol. 22, (1977), pp. 249-252.

[3] R. Palepu, J. Oliver and D. Campbell, Journal of Chem. Eng. Data, vol. 30, (1985), pp. 355.

[4] I. Nagata and J. Chem, Thermodyn, vol. 25, (1993), pp. 1281.

[5] P. S. Nikam and S. J. Kharat, Journal of Chem. Eng. Data, vol. 48, (2003), pp. 972.

[6] R. L. Gardas Ph.D. Thesis, Veer Narmad South Gujarat University, Surat, (2004).

[7] P. Paul Divakar and K. Samatha, Ind. Streams Research Journal, vol. 1, no. 9, (2011).

[8] D. Geetha and C. Rakkappan, Indian J. Phys, vol. 77B, no. 5, (2003), pp. 525.

[9] N. Prasad, Indian J pure and Appl. Ultrasonics, vol. 25, no. 25, (2003).

[10] D. Perez M, L. Segade, O. Cabeza, C. Franjo and E. Jimenez, Journal of Chem. Eng. Data, vol. 381, (2002), pp. 181.

[11] A. F. M. Barton, Chem. Rev., vol. 75, (1975), pp. 731.

[12] C. V. Suryanarayana, J. Acoust. Soc. India, vol. 4, no. 75, (1976).

[13] A. Ali and M. Tariq, J. Chem. Res. (s), vol. 4, (2006), pp. 261.

[14] J. D. Pandey, S. K. Shukla, J. Chhabra and R. Dey, Journal of Indian Chem. Soc., vol. 81, (2004), pp. 962.

[15] T. M. Aminabhavi, K. Banerjee and R. H. Balundgi, Ind. J. Chem., vol. 38A, (1999), pp. 768.

[16] S. Thirumaran and D. George, ARPN J Eng and Appl Sci., vol. 4, no. 4, (2009), pp. 1-11.

[17] J. A. Dean, Lange's Handbook of chemistry, 13th ed. New York: McGraw Hill Int., vol. 186, (1987).

[18] L. Palaniappan, Indian J. Pure Appl. Phys., vol. 40, (2002), pp. 828-830.

[19] R. Thiyagarajan, M. Suhaimi Jaafar and L. Palaniappan, Journal of Physical Science, vol. 18, no. 2, (2007), pp. 81-88.

[20] A. N. Kannappan, R. Kesavasamy and V. Ponnuswamy, ARPN J Eng. and Appl. Sci., vol. 3, no. 4, (2008), pp. 41-45.

[21] R. Thiyagarajan, M. Suhaimi Jaafar and L. Palaniappan, Journal of Physical Science, vol. 18, no. 2, (2007), pp. 81-88.

[22] R. P. Singh, C. P. Sinha, J. C. Das and P. Ghosh, Journal of Chem. Eng. Data, vol. 35, no. 1, (1990), pp. 93-97.

[23] R. K. Siwach, D. Sharma, S. K. Jangra and V. K. Sharma, Journal of Solution Chem, vol. 39, (2010), pp. 1492-1500.

[24] R. M. Pires, H. F.Costa, A. G. M. Ferreira and I. M. A. Fonseca, Journal of Chem. Eng. Data, vol. 52, (2007), pp. 1240-1245.

[25] C. S. Adgaonkar and Agnihotri, Ultrasonics, vol. 27, (1989), pp. 248.

[26] A. Ali, A. K. Nain, V. K. Sharma and S. Ahmed, Journal of Solutin Chem, vol. 42, pp. 375, (2004).

\section{Authors}

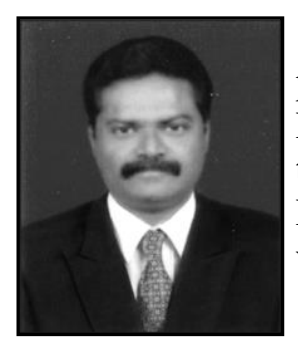

Dr. Putla Paul Divakar at present has been working as associate professor in Dept. of Physics, Sir C.R.Reddy Autonomous College, Eluru, India. He was awarded Ph.D by Andhra University in 2012 in the field of ultrasonics. He is General Assemby Member of AIRAPT, France and also Life Member of Indian Science Congress. His profile was incorporated in Marqui's Who's Who-2013, USA.

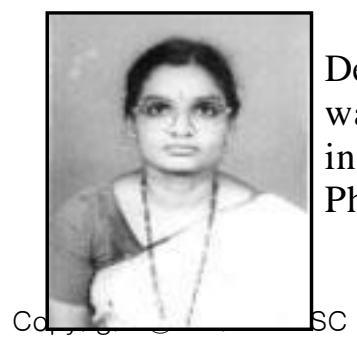

Prof. K. Samatha, has been working as a Professor in the Dept. of Physics, Andhra University, Visakhapatnam, India. She was awarded Ph.D by Andhra University, Visakhapatnam, India in 1997. She has been working in the fields of Solid State Physics and Ultrasonics. She has published 45 papers so far in 
International Journal of Advanced Science and Technology

Vol.93 (2016)

various national / international journals. Till now under her guidance $10 \mathrm{Ph}$. D Scholars and $08 \mathrm{M}$.Phil scholars were awarded with respective degrees. 